\title{
Comparison between Actual and Perceived Height of Parents of Children with Short Stature and Controls
}

\author{
Paulo F. Collett-Solberg and Pedro R. Collett-Solberg \\ Rua Saturnino de Brito 190, Lagoa 22470-030, RJ, Brazil \\ Correspondence should be addressed to Paulo F. Collett-Solberg, paulosolberg@yahoo.com \\ Received 2 December 2008; Revised 19 June 2009; Accepted 21 August 2009 \\ Recommended by Michael S. Freemark
}

Objectives. We investigate whether parents complaining of their children's short stature have misconception of their height. Methods. Parents were asked to report their own height and were then measured. We compared the difference between reported and actual height of parents of children with short stature (CSS) with that of parents coming for a well child care visit (WCC) and parents of children referred to the endocrinologist without short stature (Endo). The accuracy of reported height from short (below 25\%) and tall (above 75\%) parents was compared. Results. The CSS fathers were shorter than WCC $(P<.01)$ fathers. The CSS mothers were shorter than the Endo $(P<.01)$ and WCC $(P<.001)$ mothers. There was no difference between reported and actual height when comparing the groups based on the reason for the visit or based on the parental height. Conclusions. Parents of CSS and short parents do not have a misconception of their height.

Copyright ( 2009 P. F. Collett-Solberg and P. R. Collett-Solberg. This is an open access article distributed under the Creative Commons Attribution License, which permits unrestricted use, distribution, and reproduction in any medium, provided the original work is properly cited.

\section{Introduction}

One of the most important factors determining someone's height is the parental height [1]. Parental height is used to estimate the adult height of children, the so-called genetic target height. Physicians frequently rely on the reported height of the parents (not the measured height) to calculate, this genetic target height. Based on the genetic target height Physicians will decide if a child is growing within the family pattern or not. Several studies have previously evaluated the accuracy of reported height, but with different results [2-4]. Rona et al. looked at the reported height of parents of normal English children and found variations based on race and gender [5] while LeJarraga et al. looked at the validity of selfreported parental height at a growth clinic in Argentina and also found variations in the reported height with differences between social classes [6]. More recently Cizmecioglu et al. looked at the accuracy of reported height of parents finding that mothers reported their height with more accuracy than fathers [7]. Teitelbaum et al. told parents that the accuracy of the reported height was important for the care of the children prior to asking their heights and still found a wide range in the difference between reported and measured height [8].

Not all parents have concerns about their children's growth. We raised the question whether parents bringing their children for growth evaluation are so concerned with height that they would have a misconception of their own height. This could also affect the calculation of the genetic target height.

\section{Aims}

We compare the difference between the self-reported and measured heights of parents of children presenting to a pediatric endocrinologist office due to short stature, with two different controls. The first control group consisted of parents of children returning to a pediatric clinic for a well child care visit and the second control group consisted of parents of children referred to a pediatric endocrinologist clinic for other causes not involving short stature.

We compare the difference between the self-reported and measured heights of parents with their height below 
the 25 percentile and parents whose height is above the 75 percentile.

We compare the difference between genetic target height based on reported parental heights and the one based on the measured parental heights in the three different groups.

\section{Materials and Methods}

Patients were recruited from the office of the investigators. An Institutional Human Research Committee approved the investigation and informed consent was obtained from the subjects.

Couples presenting to the clinic were asked if they wanted to participate in the study, which was explained to them prior to asking their heights. Both parents had to be present at the time of the visit. If they consented, each one had to write down their own height and the height of the other one, to the nearest $0.1 \mathrm{~cm}$. After that each one was measured, without shoes, using a wall-mounted stadiometer graduated in $0.1 \mathrm{~cm}$. Each person was measured three times and the value used was the average between the three measurements. Height was reported in centimeters. The results were expressed as mean $\pm \mathrm{SD}$.

The differences between reported and measured heights were calculated by subtracting the measured height from the reported height (reported height-measured height). With this, a positive value means an overestimation of the height and a negative value represents an underestimation of the height.

Target height was calculated using the formula: (father's height + mother's height $\pm 13 \mathrm{~cm}$ ) $/ 2$. We add $13 \mathrm{~cm}$ for males and subtract $13 \mathrm{~cm}$ for females.

The parents were divided in three groups, based on the reason for the office visit: (1) parents of children attending the pediatrician's office for the regular well child care visit (WCC), (2) parents of children attending the pediatric endocrinologist office complaining of their child short stature (SCC), and (3) parents of children attending the pediatric endocrinology office for reasons other than short stature (Endo). The WCC control group was used to assess the knowledge of the general population of their own height. The Endo group was used to decrease the possible bias of being referred to the endocrinologist office and the knowledge of their own height.

We also compare the errors in perception based on the height of the parents. We grouped all parents and divided them based on their actual height, independently of the reason for coming to the office. For the fathers we created three groups: the ones measuring below $172 \mathrm{~cm}(25$ percentile for males), the ones between 172.1 and $181.6 \mathrm{~cm}$ and the fathers with a height above $181.7 \mathrm{~cm}$ (75 percentile for males). For the mothers we considered $158.9 \mathrm{~cm}$ as the 25 percentile and $167.7 \mathrm{~cm}$ as the 75 percentile. The percentiles were derived from NCHS 2000 data.

The assumption of normality for the data was checked by the Kolmogorv-Smirnov test. Analysis of variance followed by the Bonferroni's Multiple Comparison test was used to assess the differences between measurements and error from

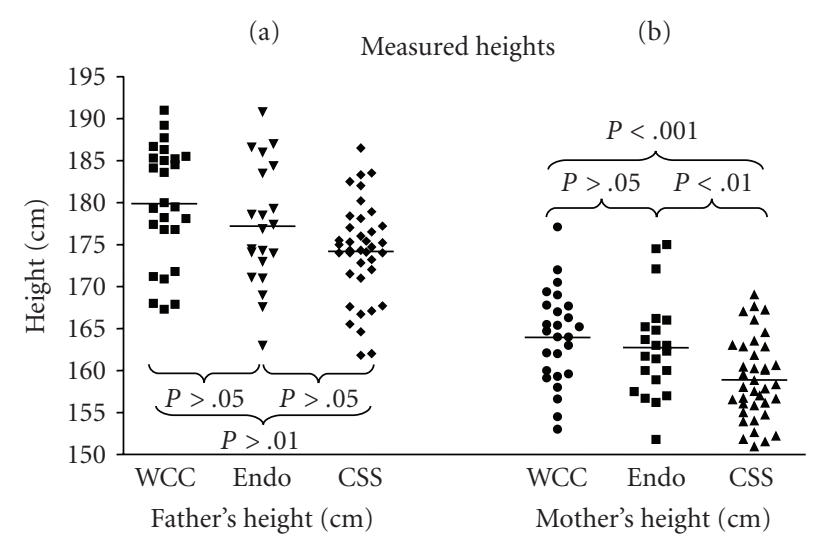

Figure 1: The height of the parents, (a) demonstrates the measured paternal heights, the mean height and the difference between paternal heights for each group, (b) demonstrates the measured maternal heights, the mean height and the difference between maternal heights for each group.

the different groups. Paired tests were used to assess the differences between actual and reported values. A $P<.05$ was considered statistically significant. The statistical program used was the GraphPad Prism -3.02.

\section{Results}

Well Child Care Group (WCC). Twenty-six couples agreed to participate. The mean maternal height was $164.0 \pm 5.5 \mathrm{~cm}$ and the mean paternal height was $179.9 \pm 6.9 \mathrm{~cm}$.

Endocrinology Group (Endo). Twenty couples agreed to participate. The mean maternal height was $163.3 \pm 5.5 \mathrm{~cm}$ and the mean paternal height was $177.2 \pm 7.4 \mathrm{~cm}$.

Short Stature Group (CSS). Thirty-eight couples agreed to participate. The mean maternal height was $158.9 \pm 4.8 \mathrm{~cm}$ and the mean paternal height was $174.2 \pm 5.8 \mathrm{~cm}$.

Figure 1 demonstrates the actual heights of the parents. The CSS fathers were shorter than the WCC fathers ANOVA $P=.0043$. The CSS mothers were shorter than the mothers in the two control groups ANOVA $P=.0004$.

Comparison between Actual Height and Reported Height. The only statistically significant difference between reported height and actual height was the difference between the mothers' height in the WCC group and their reported height by the fathers' $(P=.0409)$ as can be seen in Table 1 .

Comparison between the Difference Between Measured Heights and Reported Heights by the Mothers. Figure 2 demonstrates the difference between reported heights by the mothers and measured heights. The mean difference between the reported height and their own height for the WCC mothers was $-0.2 \pm 1.1 \mathrm{~cm}$, for the Endo mothers was $0.0 \pm$ $1.6 \mathrm{~cm}$ and for the CSS mothers was $-0.2 \pm 1.4 \mathrm{~cm}$. The difference between the reported and the measured height was 
TABLE 1: Comparison between actual and reported height $(\mathrm{cm})($ mean $\pm \mathrm{SD})$

\begin{tabular}{|c|c|c|c|}
\hline \multirow{4}{*}{ WCC } & \multirow{2}{*}{ Fathers' height $179.9 \pm 7.0$} & Reported by the father $180.1 \pm 6.8$ & $P=.505$ \\
\hline & & Reported by the mother $179.2 \pm 7.9$ & $P=.279$ \\
\hline & \multirow{2}{*}{ Mothers' height $164 \pm 5.5$} & Reported by the father $165.0 \pm 5.4$ & $P=.041^{*}$ \\
\hline & & Reported by the mother $163.7 \pm 5.7$ & $P=.327$ \\
\hline \multirow{4}{*}{ Endo } & \multirow{2}{*}{ Fathers' height $177.2 \pm 7.4$} & Reported by the father $177.3 \pm 7.9$ & $P=.754$ \\
\hline & & Reported by the mother $178.4 \pm 8.2$ & $P=.054$ \\
\hline & \multirow{2}{*}{ Mothers' height $163.3 \pm 5.5$} & Reported by the father $164.3 \pm 5.6$ & $P=.063$ \\
\hline & & Reported by the mother $163.3 \pm 5.6$ & $P=.913$ \\
\hline \multirow{4}{*}{ Short stature } & \multirow{2}{*}{ Fathers' height $174.2 \pm 5.8$} & Reported by the father $174.0 \pm 5.7$ & $P=.433$ \\
\hline & & Reported by the mother $174.2 \pm 5.9$ & $P=.903$ \\
\hline & \multirow{2}{*}{ Mothers' height $158.9 \pm 4.8$} & Reported by the father $159.4 \pm 5.9$ & $P=.383$ \\
\hline & & Reported by the mother $158.7 \pm 5.2$ & $P=.437$ \\
\hline
\end{tabular}

(a) About herself

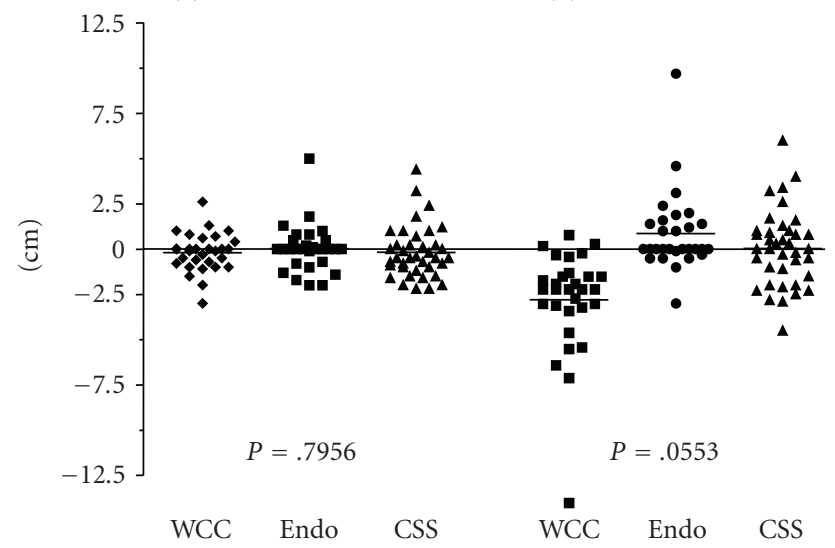

FIGURE 2: Difference, in centimeters, between heights reported by the mother and measured heights.

not statistically significant, as can be seen on Figure 2(a). Figure 2(b) demonstrates the difference between the fathers' height reported by the mothers and the fathers' actual height. The mean difference for the WCC mothers was $-0.6 \pm 3.1 \mathrm{~cm}$, for the Endo mothers was $1.2 \pm 2.6 \mathrm{~cm}$ and for the CSS mothers was $0.0 \pm 2.1 \mathrm{~cm}$. The differences were also not statistically significant.

Comparison Between the Difference Between Measured Heights and Reported Heights by the Fathers. Figure 3 demonstrates the difference between reported heights by the fathers and measured height. The mean difference between the reported height and their own height for the WCC fathers was $0.2 \pm 1.4 \mathrm{~cm}$, for the Endo fathers was $0.1 \pm 1.3 \mathrm{~cm}$ and for the CSS fathers was $-0.2 \pm 1.2 \mathrm{~cm}$. The difference between their errors was not statistically significant, as can be seen on Figure 3(a). Figure 3(b) demonstrates the difference between the mothers' height reported by the fathers and the mothers' actual height. The mean difference for the WCC fathers was $1.1 \pm 2.6 \mathrm{~cm}$, for the Endo fathers was $1.0 \pm 2.2 \mathrm{~cm}$ and for the CSS fathers was $0.5 \pm 3.3 \mathrm{~cm}$. The differences were also not statistically significant. (a) About himself

(b) About the mother

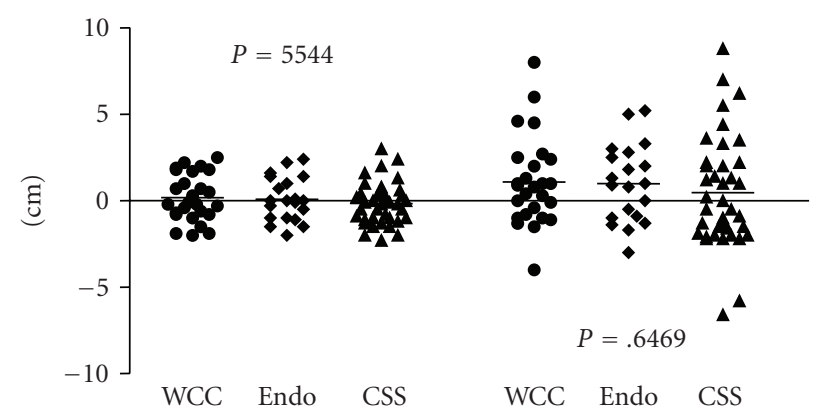

Figure 3: Difference, in centimeters, between heights reported by the father and measured heights.

Comparison between Target Height Based on Reported Height and Actual Target Height. Figure 4 demonstrates the difference between target height based on reported height and actual target height. The first column of each group (father) demonstrates the difference $(\mathrm{cm})$ between the target height based on data reported by the fathers and the actual target height based on the values obtained at the time of the visit. The second column of each group (mother) demonstrates the difference $(\mathrm{cm})$ between the target height based on data reported by the mothers and the actual height. The third column (both) demonstrates the difference $(\mathrm{cm})$ between the target height based on the fathers' and mothers' reports of their own height and the actual target height. There was no statistically significant difference between any of the reported target height and the actual target height. Most of the difference between measured and reported target height stayed between -2 and $+3 \mathrm{~cm}$, as can be seen with the dotted lines.

Comparison between the Errors Reported by Short (Height Below the 25 Percentile) Parents, Average Parents and Tall Parents (Height Above the 75 Percentile). There were, in the whole group, 22 fathers with heights below the 25 percentile and 23 above the 75 percentile. For the mothers, there were 30 with heights below the 25 percentile and 10 above the 75 percentile. There was no statistical difference between the 


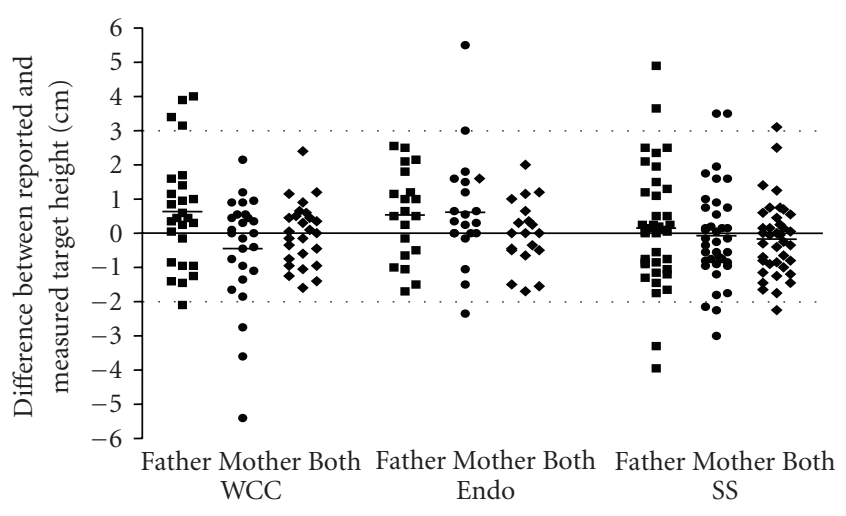

Figure 4: Difference, in centimeters, between target heights calculated with reported values and the measured target height.

errors in the reported height when groups were divided based on the actual height, data not shown.

\section{Discussion}

The accuracy of reported height has been previously published. In our study, there was no statistically significant difference between reported height and measured height in most groups. The exception was the fathers' report of the mothers' height in the WCC group.

Similar to our study that compared the reported height of controls and of parents with questions about growth, Beyer et Doerr [9] published that mothers of children with familial short stature reported to be taller than in reality although the mean difference was $1.9 \mathrm{~cm}$. In that study fathers were not present and consequently their own report, their report about the mothers' height and the mothers' report of the fathers' height were not investigated. Teitelbaum et al. found that fathers reported to be taller than they really were [8]. In that work, parents were specifically alerted that their reported height was important for the care of the children in an attempt to increase accuracy. The difference between measured and reported height in our study was not statistically significant. Maybe, in the studies by Teitelbaum et al. [8] and by Beyer and Doerr [9], parents thought that reporting themselves taller would increase the concern of the doctor with their children's growth and care.

Our study had one new aspect in the investigation of accuracy of reported height. We informed the parents, before they reported their height, that they were going to be measured; and by knowing that they would be measured, we estimate that we encouraged them to be as accurate as possible. The accuracy of the reported height was similar between the groups, demonstrating that parents bringing their children for growth evaluation do not have a misconception of their own height. Even though they were shorter than the control parents, the accuracy of their reported height was similar.

We also showed no difference in the accuracy of reported height by "short" parents with the accuracy of reported height by "tall" parents.
The difference between actual target height and reported target height was not statistically significant but showed a wide variation, with most of the errors ranging between -2 and $+3 \mathrm{~cm}$. If the parents did not know they were going to be measured would their reported value be as accurate? Since the objective of this work was to assess the parents own perception of their (and the other parent) height, our study achieved its objective although we cannot say that the information given by parents in the pediatrician/pediatric endocrinologist office is going to be as accurate.

With this study we demonstrated that parents bringing their children to a pediatric endocrinology office are shorter than the two controls groups but they were as accurate as the control group in their knowledge of their own height.

\section{Conclusion}

Differences between reported heights and measured heights are sometimes observed, although, in the majority of cases, the reported heights were within $3 \mathrm{~cm}$ of the actual height. Importantly, parents of short children do not have a misconception of their own heights, when compared with controls.

\section{Acknowledgments}

This work was partially presented at the ESPE/LWPES 7th joint meeting Paediatric Endocrinology, Lyon, France, 2005. Grant support: Genentech Foundation for Growth and Development (PFCS). The authors would like to thank Dr. Michael Freemark and Dr. Charles Stanley for the help in developing this project and preparing the manuscript.

\section{References}

[1] R. Rapaport and D. A. Bowlby, "Clinical aspects of growth and growth disorders," in Pediatric Endocrinology, O. H. Pescovitz and E. A. Eugster, Eds., p. 173, Lippincott Williams \& Wilkins, Philadelphia, Pa, USA, 2004.

[2] P. Pirie, D. Jacobs, R. Jeffery, and P. Hannan, "Distortion in self-reported height and weight data," Journal of the American Dietetic Association, vol. 78, no. 6, pp. 601-606, 1981.

[3] A. L. Stewart, "The reliability and validity of self-reported weight and height," Journal of Chronic Diseases, vol. 35, no. 4, pp. 295-309, 1982.

[4] M. Palta, R. J. Prineas, R. Berman, and P. Hannan, "Comparison of self-reported and measured height and weight," American Journal of Epidemiology, vol. 115, no. 2, pp. 223-230, 1982.

[5] R. J. Rona, S. Chinn, and R. Manning, "The validity of reported parental height in inner city areas in England," Annals of Human Biology, vol. 16, no. 1, pp. 41-44, 1989.

[6] H. LeJarraga, M. Laspuir, and P. Adamo, "Validity of reported parental height in outpatient growth clinics in Buenos Aires city," Annals of Human Biology, vol. 22, no. 2, pp. 163-166, 1995.

[7] F. Cizmecioglu, A. Doherty, W. F. Paterson, D. Young, and M. D. Donaldson, "Measured versus reported parental height," Archives of Disease in Childhood, vol. 90, no. 9, pp. 941-942, 2005. 
[8] J. E. Teitelbaum, S. Koreen, K. Hightower, R. Rajaraman, and J. Jaeger, "Inaccuracy of stated versus measured parental heights," Clinical Pediatrics, vol. 44, no. 4, pp. 339-341, 2005.

[9] R. Ch. Beyer and H. G. Doerr, "Observations of reported and measured heights of mothers of short statured children," Annals of Human Biology, vol. 25, no. 4, pp. 387-390, 1998. 